\title{
Author Correction: Neoadjuvant immune checkpoint blockade in high-risk resectable melanoma
}

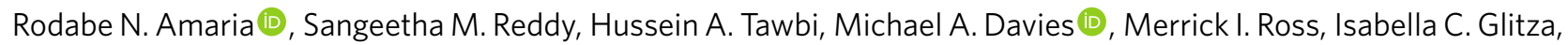
Janice N. Cormier, Carol Lewis, Wen-Jen Hwu, Ehab Hanna, Adi Diab, Michael K. Wong, Richard Royal, Neil Gross (D), Randal Weber, Stephen Y. Lai, Richard Ehlers, Jorge Blando, Denái R. Milton, Scott Woodman, Robin Kageyama, Daniel K. Wells, Patrick Hwu, Sapna P. Patel, Anthony Lucci, Amy Hessel, Jeffrey E. Lee, Jeffrey Gershenwald, Lauren Simpson, Elizabeth M. Burton, Liberty Posada, Lauren Haydu, Linghua Wang (D), Shaojun Zhang, Alexander J. Lazar (1), Courtney W. Hudgens (), Vancheswaran Gopalakrishnan, Alexandre Reuben (D), Miles C. Andrews, Christine N. Spencer, Victor Prieto, Padmanee Sharma, James Allison, Michael T. Tetzlaff and Jennifer A. Wargo

Correction to: Nature Medicine https://doi.org/10.1038/s41591-018-0197-1, published online 08 October 2018.

In the version of this article originally published, there was an error in Fig. 2b. RECIST ORR and pCR were both listed as $25 \%$. RECIST ORR was actually $73 \%$, and pCR was $45 \%$. Also, an author's name was incorrect in the author list. Danny K. Wells should have been listed as Daniel K. Wells. The errors have been corrected in the print, HTML and PDF versions of this article.

\section{Publisher Correction: Single-cell profiling of breast cancer T cells reveals a tissue- resident memory subset associated with improved prognosis}

Peter Savas (D), Balaji Virassamy, Chengzhong Ye, Agus Salim, Christopher P. Mintoff, Franco Caramia, Roberto Salgado, David J. Byrne, Zhi L. Teo, Sathana Dushyanthen, Ann Byrne, Lironne Wein, Stephen J. Luen, Catherine Poliness, Sophie S. Nightingale, Anita S. Skandarajah, David E. Gyorki, Chantel M. Thornton, Paul A. Beavis, Stephen B. Fox ID, Kathleen Cuningham FoundationConsortium for Research into Familial Breast Cancer (kConFab), Phillip K. Darcy, Terence P. Speed, Laura K. Mackay (D), Paul J. Neeson and Sherene Loi ${ }^{\mathbb{D}}$

Correction to: Nature Medicine https://doi.org/10.1038/s41591-018-0078-7, published online 25 June 2018.

In the version of this article originally published, the institution in affiliation 10 was missing. Affiliation 10 was originally listed as Department of Surgery, Royal Melbourne Hospital and Royal Womens' Hospital, Melbourne, Victoria, Australia. It should have been Department of Surgery, Royal Melbourne Hospital and Royal Womens' Hospital, University of Melbourne, Melbourne, Victoria, Australia. The error has been corrected in the HTML and PDF versions of this article.

\section{Publisher Correction: Clinical activity and molecular correlates of response to atezolizumab alone or in combination with bevacizumab versus sunitinib in renal cell carcinoma}

David F. McDermott, Mahrukh A. Huseni, Michael B. Atkins, Robert J. Motzer, Brian I. Rini, Bernard Escudier, Lawrence Fong, Richard W. Joseph, Sumanta K. Pal, James A. Reeves, Mario Sznol, John Hainsworth, W. Kimryn Rathmell, Walter M. Stadler, Thomas Hutson, Martin E. Gore, Alain Ravaud, Sergio Bracarda, Cristina Suárez, Riccardo Danielli, Viktor Gruenwald, Toni K. Choueiri, Dorothee Nickles, Suchit Jhunjhunwala, Elisabeth Piault-Louis, Alpa Thobhani, Jiaheng Qiu, Daniel S. Chen, Priti S. Hegde, Christina Schiff, Gregg D. Fine and Thomas Powles

Correction to: Nature Medicine https://doi.org/10.1038/s41591-018-0053-3, published online 4 June 2018.

In the version of this article originally published, there was an error in Fig. $2 \mathrm{n}$. The top line of the HR comparison chart originally was Atezo + bev vs sun. It should have been Atezo + bev vs atezo. The error has been corrected in the HTML and PDF versions of this article. 